follow-up appointments generated or discharge rates from clinic. During the study period, nurse led care resulted in increased resource use compared with consultant led care - but could be partly explained by the greater proportion of patient seen in the 'suspected cancer' pathway. No adverse outcomes or missed diagnoses were observed over an 18 month follow up period. Our study would support the role of specialist nursing managing unselected patients in gastroenterology clinics.

Disclosure of Interest None Declared.

\section{PTH-031 AN INCREASING DEMAND FOR ENHANCED SEDATION ENDOSCOPY: A SINGLE CENTRE EXPERIENCE}

${ }^{1} \mathrm{D}$ Joshi*, ${ }^{1} \mathrm{M}$ Austin, ${ }^{2} \mathrm{~T}$ Bate, ${ }^{1} \mathrm{~S}$ Cairns. 'Digestive Disease Centre, Brighton and Sussex University Hospital, Brighton, UK; ${ }^{2}$ Department of Anaesthesia, Brighton and Sussex University Hospital, Brighton, UK

\subsection{6/gutjnl-2014-307263.477}

Introduction The demand for enhanced sedation endoscopy (ESE) appears to be increasing due to increasingly challenging diagnostic and therapeutic procedures. BSG Working Party guidance were issued in 2011. ${ }^{1}$ Our aim is to describe a single centre experience and highlight the importance of providing such a service.

Methods Retrospective review of all patients undergoing elective ESE at Brighton and Sussex University Hospital (BSUH) from March 2012 to March 2013. Cases were performed in the endoscopy department and in a day surgery theatre. Patients were identified using the Unisoft endoscopy program and day theatre list records. Patient records were reviewed in addition to endoscopy reports. Data collection included patient demographics, indication for procedure, procedure length, success of procedure if previously failed and complications. Data presented as median with range.

Results A total of 89 procedures (OGD x 25 (28\%), colonoscopy x $28(31 \%)$, flexible sigmoidoscopy x $2(2 \%)$, ERCP x 24 $(27 \%)$, OGD/colonoscopy x $10(9 \%))$ in 79 patients were performed. Median age 53 years (22-75 years), weight $77.5 \mathrm{~kg}$ (52$126 \mathrm{~kg}$ ), BMI 26 (22-48), female 60\%, ASA 2 (1-4). Indications for ESE included a previously poorly tolerated procedure $(38 \%$, $\mathrm{n}=34)$, co-morbidities $(24 \%, \mathrm{n}=21)$, patient choice $(20 \%, \mathrm{n}$ $=18$ ), previously failed procedure $(9 \%, \mathrm{n}=10)$ and likely long procedure $(7 \%, \mathrm{n}=6) .80$ patients received a combination of propofol/ fentanyl sedation whilst 9 patients required a full general anaesthetic. ESE was delivered by a designated anaesthetist. Median duration of procedure was $35 \mathrm{~min}$ (10-65 min). There were no endoscopic related complications. 1 patient developed hypotension requiring intravenous fluids and was admitted for observations overnight and 1 patient developed bronchospasm post extubation requiring intravenous steroid and nebulisers but did not require admission. The use of ESE resulted in the successful completion of all endoscopic procedures.

Conclusion Review of our referrals demonstrates an increasing demand of ESE. Our service initially began as an ad hoc list but now is weekly. ESE appears to be a safe, time efficient and reduces the requirement for repeat procedures.

\section{REFERENCE}

1 Guidance for th, UKe use of propofol sedation for adult patients undergoing ERCP and other complex upper GI endoscopy procedures, April 2011. RCoA and BSG working party

Disclosure of Interest None Declared.

\section{PTH-032 GUT HORMONE SCREENING FOR GASTROENTEROPANCREATIC NEUROENDOCRINE TUMOURS - A QUALITY IMPROVEMENT PROJECT}

${ }^{1}$ D Mansour*, ${ }^{2}$ T Lee. ' Gastroenterology, Gateshead Queen Elizabeth Hospital, Newcastle Upon Tyne, UK; ${ }^{2}$ Gastroenterology, Northumbria Healthcare Trust, North Tyneside, UK

\subsection{6/gutjnl-2014-307263.478}

Introduction BSG guidelines advise that, in patients presenting with symptoms suspicious of gastroenteropancreactic neuroendocrine tumour (NET), baseline gut hormone $(\mathrm{GH})$ tests should include Chromogranin A (CgA) and urinary 5HIAA. Other specific biochemistry should be requested depending on the syndrome suspected. We reviewed the use of gut hormone screening in a North East England Trust and examined the association between positive results and NET diagnosis.

Methods We reviewed all GH screens requested between July 2012 and June 2013. The following data were collected: specialty of requesting physician, indication, results and clinical outcome. We compared results of GH screens (CgA and then other $\mathrm{GHs}$ ) with the diagnosis of NET to calculate specificity. Finally, we looked at all NET diagnosed in the trust over the same period, reviewing GH levels in those tested following diagnosis, in order to calculate sensitivity. Financial implications of different GH testing strategies were assessed using these results.

Results Of a total 51 requests for gut hormone screens, 21 were made by gastroenterologists and 8 by surgeons. 19 requests in total were made for investigation of diarrhoea, 12 for upper GI symptoms/peptic ulcers, 5 following positive histology or lesions on imaging and 15 for other symptoms. A total of $459 \mathrm{GH}$ were tested at a cost of $£ 315$ per patient. $32 / 51$ patients had normal CgA levels, none of which went on to be diagnosed with NET. 19 had a raised CgA of which 2 were already known to have NET but no new NET were found (specificity 65.31\%). Patients with diarrhoea had a particularly high false positive rate (7/ $19=37 \%$ ). Of 18 patients newly diagnosed with NETs in the trust, 5 had GHs tested (all following diagnosis)-3/5 had raised CgA (cost $£ 44$ per patient), a sensitivity of $60 \%$ (this increased to $80 \%$ when combined with u5HIAA testing). Measuring other gut hormones only marginally increased sensitivity but greatly reduced specificity of the screening.

Conclusion Gut hormone screening was not being performed in line with BSG recommendations in our Trust, leading to excessive numbers of tests being performed with low sensitivity and specificity. We worked with the trust biochemistry department to clarify the indications for $\mathrm{GH}$ testing and rationalise the screening test performed. We now offer an 'endocrine diarrhoea screen' of CgA and u5HIAA. Other hormones are measured only if a specific syndrome is suspected/in patients with known history/family history of NET, representing a cost saving of $£ 271$ per patient. It is envisaged that this change in practice will save the trust at least $£ 12,000$ per annum whilst improving clinicians` decision making around testing for NET.

Disclosure of Interest None Declared.

\section{PTH-033 A SPECIALIST IRON DEFICIENCY ANAEMIA CLINIC SIGNIFICANTLY REDUCES THE NEED FOR SECONDARY CARE FOLLOW UP}

E Taylor*, J Campbell, M Donnelly, J Hebden. Gastroenterology, Sheffield Teaching Hospitals, Sheffield, UK

10.1136/gutjnl-2014-307263.479 
Introduction BSG guidelines provide clear recommendations for the investigation and management of iron deficiency anaemia (IDA). The algorithm lends itself to a 'one stop' new patient clinic in secondary care with further follow-up in primary care. We set up a dedicated IDA clinic aiming to streamline patients' management and reduce unnecessary follow-up visits.

Methods A 'one stop' IDA clinic was set up and run by a single gastroenterologist from November 2013. Data was collected prospectively for the first 60 patients referred with IDA (Group A). Patients without confirmed IDA (10, [17\%]) were analysed separately. A second group of proven IDA referrals seen in unselected gastroenterology clinics in 2011 was identified (Group B). Rates of clinic follow up were recorded and the two groups compared. Additional data collected included demographics, haemoglobin, MCV, ferritin and other iron indices (Fe/TIBC) as well as radiology and endoscopy reports. Iron deficiency was defined as isolated microcytosis and/or low ferritin.

Results Fifty patients fulfilled diagnostic criteria for IDA in group A (35 female, median age 69.0, range 35-91). Group B comprised 50 IDA patients (28 female, median age 72.0, range 38-83). All patients in Group A were seen by a single, UKconsultant compared to $80 \%$ seen by consultants in Group B.

Four patients were diagnosed with colorectal cancers in group A. Two patients were diagnosed with oesophago-gastric tumours and 4 with colorectal cancers in group B. One new diagnosis of coeliac disease was made in each group. Group A contained five females with gynaecological pathology responsible for IDA (1 advanced cervical cancer and 4 menorrhagia).

Despite confirmation of IDA with a low ferritin, $11(22 \%)$ in Group A and $6(12 \%)$ in Group B had undergone additional serum iron/TIBC measurements before referral. Only 3/17 $(18 \%)$ iron studies were congruent with the ferritin result.

Significantly more patients in Group A (94\%) were discharged back to primary care after their initial consultation and investigations compared to Group B $(26 \%, \mathrm{p}<0.0001)$.

Conclusion A specialist IDA clinic leads to appropriate discharge to primary care virtually eliminating secondary care follow up. Clinical assessment allows identification of a significant proportion of non-IDA referrals requiring alternative management. Measurement of serum iron/TIBC should be discouraged. Disclosure of Interest None Declared.

\section{PTH-034 THE SAFE AND EFFECTIVE TREATMENT OF IRON DEFICIENCY WITH IRON ISOMALTOSIDE 1000 - CLINICAL EXPERIENCE FROM A UK GASTROENTEROLOGY UNIT}

'E Williams*, 'L Craven, 'S Surgenor, ${ }^{2} \mathrm{U}$ Bapat, ${ }^{3} \mathrm{C}$ Strom, ${ }^{1} \mathrm{~J}$ Snook. ${ }^{1}$ Poole Hospital Foundation Trust, Poole, UK; ${ }^{2}$ Pharmacosmos, Thame, UK; ${ }^{3}$ Pharmacosmos, Holbaek, Denmark

\subsection{6/gutjnl-2014-307263.480}

Introduction Parenteral iron has been used increasingly over the last decade to treat specific patient groups with iron deficiency (ID) anaemia. There is, however, limited evidence of safety and efficacy in all patients with ID, particularly those with underlying malignancy or inflammation.

Methods Data from all outpatients receiving iv iron isomaltoside (Monofer) for confirmed ID between 1st April and 31st December 2012 at our UK District General Hospital was analysed, regardless of underlying diagnosis or comorbidity. Patients received their infusions under the care of Gastroenterology on our Medical Investigations Unit, a 7-day nurse led unit that works extended hours and is located within the main hospital.

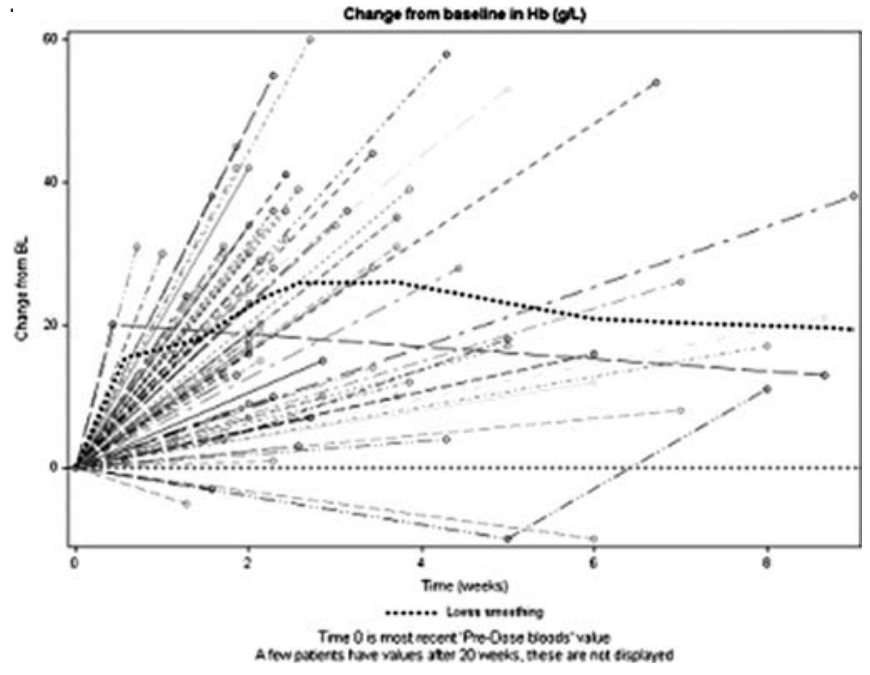

Abstract PTH-034 Figure 1

ID was defined by ferritin $<24 \mathrm{mcg} / \mathrm{l}$, transferrin saturation index (TSAT) $<15 \%$ or a blood film compatible with ID. Anaemia was defined as a low haemoglobin concentration $(\mathrm{Hb})$ (men $<130 \mathrm{~g} / \mathrm{l}$, women $<115 \mathrm{~g} / \mathrm{l})$. Data was collected for each patients first treatment within the study period only, albeit 1 or 2 infusions. The dose of parenteral iron was at the discretion of the referring Consultant. All patients had post infusion bloods, $75(88 \%)$ within 4 weeks. Data on the cause of ID and significant comorbidity was collected.

Results 85 patients received monofer infusions for confirmed ID over the study period, with 80 of these having ID anaemia. Referrals were from a range of medical, surgical and oncological specialities. 55 patients (65\%) were female and the age range was 18-92 years (median 73 years). Baseline bloods (median and interquartile range (IQR)) were $\mathrm{Hb} 90 \mathrm{~g} / \mathrm{l}$ (80-99), Mean Cell Volume 81 fl (72-88), Mean Cell Haemoglobin 24.8 pg (22.228.0), ferritin $15 \mathrm{mcg}(9-24)$ and TSAT 9\% (5-15).

Most patients had extensive comorbidity; 29(34\%) had active malignancy and 44(77\%) of those without malignancy had active inflammation. The median iron dose was $14.6 \mathrm{mg} / \mathrm{kg}$ (IQR 11.2-17.5). No significant adverse reactions occurred and all patients received the full infusion.

Post infusion bloods demonstrated a median increase in $\mathrm{Hb}$ of $23 \mathrm{~g} / \mathrm{l}$ (Figure 1) with $52 \%$ having a $\mathrm{Hb}$ increase of $>20 \mathrm{~g} / \mathrm{l}$. $\mathrm{The} \mathrm{Hb}$ rise was significantly higher in individuals with a lower baseline $\mathrm{Hb}$; regression slope of baseline against change in $\mathrm{Hb}=$ $-5.9 \mathrm{Hb}, \mathrm{p}<0.0001$.

Conclusion Treatment of ID anaemia with parenteral iron is safe and effective in all patients including those with cancer or active inflammation. A rapid rise in $\mathrm{Hb}$ within 4 weeks is seen in many patients particularly where the baseline $\mathrm{Hb}$ is low.

Disclosure of Interest None Declared.

\section{PTH-035 PULMONARY MISPLACEMENT OF NASOGASTRIC TUBES: A 2 YEAR AUDIT OF SERVICE DELIVERY, FEEDBACK INTERVENTION AND PATIENT OUTCOME}

${ }^{1} \mathrm{~F}$ Mansour*, 'M Virta, ${ }^{2} \mathrm{P}$ Neild, ${ }^{3} \mathrm{~T}$ Marshall. 'St George's Medical School, St George's University of London, London, UK; ' ${ }^{2}$ Gastroenterology, St George's NHS Trust, London, UK; ${ }^{3}$ Nutrition, St George's NHS Trust, London, UK

\subsection{6/gutjnl-2014-307263.481}

Introduction Although nasogastric feeding will continue to be the preferred method of assisted nutrition, after oral intake, better 